\title{
MicroRNA-34a-5p serves as a tumor suppressor by regulating the cell motility of bladder cancer cells through matrix metalloproteinase-2 silencing
}

\author{
KUANG-YU CHOU ${ }^{1,2}$, AN-CHEN CHANG $^{3}$, TE-FU TSAI ${ }^{1,2}$, YI-CHIA LIN $^{1,2}$, HUNG-EN CHEN $^{1}$, \\ CHAO-YEN HO ${ }^{1,4}$, PO-CHUN CHEN ${ }^{3,5}$ and THOMAS I-SHENG HWANG ${ }^{1,2,6}$
}

\begin{abstract}
${ }^{1}$ Division of Urology, Department of Surgery, Shin Kong Wu Ho-Su Memorial Hospital, Taipei 11102; ${ }^{2}$ Division of Urology, School of Medicine, Fu-Jen Catholic University, New Taipei 242062; ${ }^{3}$ Translational Medicine Center, Shin Kong Wu Ho-Su Memorial Hospital, Taipei 11102; ${ }^{4}$ Institute of Traditional Medicine, School of Medicine, National Yang-Ming University, Taipei 112304; ${ }^{5}$ Department of Biotechnology, College of Health Science, Asia University, Taichung 41354; ${ }^{6}$ Department of Urology, Taipei Medical University, Taipei 110301, Taiwan, R.O.C.
\end{abstract}

Received May 21, 2020; Accepted November 9, 2020

DOI: $10.3892 /$ or.2020.7910

\begin{abstract}
Bladder cancer (BC), a common urologic cancer, is the fifth most frequently diagnosed tumor worldwide. hsa-miR-34a displays antitumor activity in several types of cancer. However, the functional mechanisms underlying hsa-miR-34a in BC remains largely unknown. We observed that hsa-mir-34a levels were significantly and negatively associated with clinical disease stage as well as regional lymph node metastasis in human BC. In a series of in vitro investigations, overexpression of hsa-miR-34a inhibited cell migration and invasion in BC cell lines 5637 and UMUC3 as detected by Transwell assays. We further found that hsa-miR-34a inhibited cell migration and invasion by silencing matrix metalloproteinase-2 (MMP-2) expression and thus interrupting MMP-2-mediated cell motility. Our analysis of BC datasets from The Cancer Genome Atlas database revealed a negative correlation between hsa-miR-34a and MMP-2. Moreover, higher MMP-2 protein expression was observed in the $\mathrm{BC}$ tissues when compared with that noted in the normal tissue. MMP-2 levels were also significantly associated with clinical disease stage and poor survival rate in human BC. These findings indicate that MMP-2 plays a critical role in regulating $\mathrm{BC}$ progression. Therefore, hsa-miR-34a is a
\end{abstract}

Correspondence to: Dr Thomas I-Sheng Hwang, Division of Urology, Department of Surgery, Shin Kong Wu Ho-Su Memorial Hospital, 95 Wenchang Road, Shilin, Taipei 11102, Taiwan, R.O.C.

E-mail: thomashwang0820@gmail.com

Dr Po-Chun Chen, Translational Medicine Center, Shin Kong Wu Ho-Su Memorial Hospital, 95 Wenchang Road, Shilin, Taipei 11102, Taiwan, R.O.C.

E-mail: blibra1002@gmail.com

Key words: bladder cancer, microRNA-34a-5p, migration, invasion, MMP-2 promising treatment to target MMP-2 for the prevention and inhibition of cell migration and invasion in $\mathrm{BC}$.

\section{Introduction}

Bladder cancer (BC) is the fifth most commonly diagnosed tumor worldwide and the second leading cause of death in patients with genitourinary tract malignancies (1). Annually, approximately 430,000 new BC cases and 165,000 cancer deaths are reported globally, according to the International Agency for Research on Cancer and the World Health Organization (2). The vast majority (an estimated $80 \%$ ) of BC is diagnosed as nonmuscle invasive (NMIBC), also known as superficial $\mathrm{BC}$, and the remaining $20 \%$ are muscle (i.e., muscularis propria)-invasive BC (MIBC) (3). The overall survival rate of patients diagnosed with NMIBC is high, although $30-50 \%$ of NMIBC will recur after transurethral resection and $10-20 \%$ will progress to MIBC (4). MIBC is responsible for the vast majority of $\mathrm{BC}$-specific deaths, and approximately $50 \%$ of patients with MIBC present a higher incidence of distant metastasis than those with NMIBC at the time of diagnosis (5). Currently, transurethral resection of a bladder tumor (TURBT) and intravesical chemotherapy are still considered the standard of care for bladder cancer. However, not all patients are suitable for chemotherapy. Viable treatment alternatives are warranted in the treatment of BC.

Tumor metastasis is a complex and continuous multistep process encompassing local adhesion, migration, and invasion that also accompany a variety of proteases, particularly matrix metalloproteinases (MMPs) (6). MMPs, a group of calcium $\left(\mathrm{Ca}^{2+}\right)$ - and zinc $\left(\mathrm{Zn}^{2+}\right)$-dependent endopeptidases, are responsible for the tissue remodeling and degradation of the extracellular matrix (ECM) leading to tumor cell migration and invasion in the metastatic environment $(7,8)$. In total, 24 genes encoding MMPs in humans have been identified and many are implicated in cancer (9). For instance, MMP-1, MMP-3, and MMP-9 expression levels are high in human chondrosarcoma cells, and MMP-9 is positively correlated with clinical outcome 
parameters in chondrosarcoma (10). The secretion of MMP-2 and MMP-9 is elevated in various types of human cancers, and their expression is closely related to clinical staging, lymph node metastasis, and poor prognosis (11-13).

MicroRNAs (miRNAs/miRs), consisting of approximately 22 nucleotides, are non-coding RNAs (14) that bind to target mRNAs and suppress protein translation by either blocking the translation or destabilizing mRNA levels (15). Many miRNAs are classified as either tumor-suppressive (TS) miRNAs or oncogenic miRNAs, also known as oncomirs (16). miR-34a is a TS miRNA, and its expression is epigenetically silenced in several human cancers $(17,18)$. Expression of hsa-miR-34a stimulates cell apoptosis, cell cycle arrest, cellular senescence, epithelial-mesenchymal transition (EMT) repression, and impedes cell proliferation in cancer stem cells (19). Moreover, hsa-miR-34a is downregulated in BC tissues and has been demonstrated to inhibit migration and invasion by silencing Notch1 (20) or CD44 (21). Overexpression of hsa-miR-34a may also inhibit cell migration and invasion and thus suppress metastasis in hepatocellular carcinoma (22). In breast cancer, low hsa-miR-34a expression was found to advance cell invasion in vitro and distant metastasis in vivo by directly silencing proto-oncogene Fos-related antigen-1 (Fra-1) expression (23). These findings highlight hsa-miR-34a as a potential therapeutic target and a clinical biomarker of cancer progression and metastasis.

The present study revealed that hsa-miR-34a expression is negatively associated with clinical disease stage, regional lymph node metastasis, and overall survival rate in patients with BC. In vitro evidence revealed that hsa-miR-34a suppressed MMP-2 expression, leading to inhibition of BC cell motility. Another critical clinical characteristic of MMP-2 was its higher expression in late stage than in early stage BC. Moreover, high expression of MMP-2 was found to be significantly associated with a lower survival rate for patients with BC. This study provides insight into the mechanisms underlying hsa-miR-34a-mediated cell migration and invasion through MMP-2 silencing in $\mathrm{BC}$.

\section{Materials and methods}

Cell culture. Human BC cell lines (5637 and UMUC3) and human bladder epithelial cell line (SV-HUC-1) were obtained from Bioresource Collection and Research Center (Hsinchu, Taiwan) and incubated at $37^{\circ} \mathrm{C}$ under $5 \% \mathrm{CO}_{2}$. SV-HUC-1 cells were cultured in F-12 medium (Gibco; Thermo Fisher Scientific, Inc.). 5637 and UMUC3 cells were cultured in RPMI-1640 medium (Gibco; Thermo Fisher Scientific, Inc.). All culture media were supplemented with $10 \%$ fetal bovine serum (FBS), 2 mM GlutaMAX-1, $100 \mathrm{U} / \mathrm{ml}$ penicillin, and $100 \mu \mathrm{g} / \mathrm{ml}$ streptomycin.

hsa-miR-34a stable clones in BC cells. MicroRNA expression vectors were constructed by annealing a paired oligonucleotide consisting of mature hsa-miR-34a-5p sequences and cloning it into a small-RNA expression vector (pSM-vector), as previously described (24). Culture cells with $70 \%$ confluence were transfected with pSM-34a-5p in 6-well plates for $24 \mathrm{~h}$ prior to the administration of G418 selection antibiotic. The transfection was performed using polymer-based transfection reagents
(Ultra293, GeneDirex, Taipei, Taiwan) according to manufacturer's instructions. The cell viability of each transfected cell line was tested to investigate whether miRNA disrupted BC cell proliferation.

Transfection of hsa-miR-34a mimic and inhibitor. MISSION synthetic negative control (NC) mimic (5'-GGUUCGUAC GUACACUGUUCA-3'), hsa-miR-34a-5p mimic (5'-UGGCAG UGUCUUAGCUGGUUGU-3'), NC inhibitor (5'-GGUUCG UACGUACACUGUUCA-3') and hsa-miR-34a-5p inhibitor (5'-ACAACCAGCUAAGACACUGCC-3') were purchased from Sigma-Aldrich/Merck KGaA. Cells were seeded in 6-well dishes with $2 \mathrm{ml}$ culture medium. Viromer ${ }^{\circledR}$ BLUE (Lipocalyx GmbH, Germany), miRNA transfection reagent, was used to transfect hsa-miR-34a mimic (100 nM) or inhibitor $(100 \mathrm{nM})$ in cells for $24 \mathrm{~h}$. Cell samples were then evaluated for MMP-2 expression and the ability of cell migration and invasion by Western blot analysis and Transwell migration assay, respectively.

Luciferase reporter assay. Wild-type (5'...CACUGCC....3') and mutant (5'...GAGAGGC...3') plasmids of human MMP-2 3'-UTR (untranslated region) containing the miR-34a-5p binding site were constructed in pmiR-GLO (Promega Corp.) by MDBio, Inc. (Taipei, Taiwan). BC cells were seeded in 6-well dishes and transfected with the plasmid $(1 \mu \mathrm{g} / \mu \mathrm{l})$, using Viromer ${ }^{\circledR}$ RED (Lipocalyx $\mathrm{GmbH}$ ), as per manufacturer's protocol. After $24 \mathrm{~h}$ of transfection, cell lysates were harvested and the activities of firefly and Renilla luciferase were detected using Dual-Luciferase kit (Promega Corp.). Firefly luciferase activity was normalized to Renilla luciferase activity.

Western blot analysis. Total proteins were extracted from BC cell lines. The samples containing $20 \mu \mathrm{g}$ of total protein were electrophoresed on $12 \%$ sodium dodecyl sulfate-polyacrylamide gel electrophoresis (SDS-PAGE) gels and proteins were transferred to Immobilon polyvinyldifluoride membranes. Bovine serum albumin (4\%) was used to block membranes for $1 \mathrm{~h}$ at room temperature; the membranes were then probed with primary anti-MMP-2 (dilution 1:3,000; GeneTex; cat. no. GTX104577), anti-b-actin (dilution 1:5,000; Merck; cat. no. MAB1501) and anti-GAPDH (dilution 1:5,000; Abcam; cat. no. ab8245) antibodies at $4^{\circ} \mathrm{C}$ overnight. The membranes were incubated with the appropriate horseradish peroxidase (HRP)-conjugated anti-rabbit antibody (dilution 1:3,000; Cell Signaling; cat. no. 7074S) or anti-mouse antibody (dilution 1:3,000; Sigma-Aldrich/Merck KGaA; A9044) at $37^{\circ} \mathrm{C}$ for $1 \mathrm{~h}$. Enhanced chemiluminescence was then added to the blots, which were imaged using a ChemiDoc-It Imaging System (UVP Inc.).

Quantitative real-time polymerase chain reaction. Total RNA extracted from BC cells was reverse transcribed and subjected to quantitative real-time polymerase chain reaction (qPCR) to detect MMP-2 and GAPDH as previously described (25).

Migration and invasion assays. Transwell inserts in 24-well dishes $(8-\mu \mathrm{m}$ pore size; Costar) were used to perform cell migration and invasion. For invasion assays, Transwell inserts were precoated with $30 \mu \mathrm{l}$ Matrigel basement membrane 

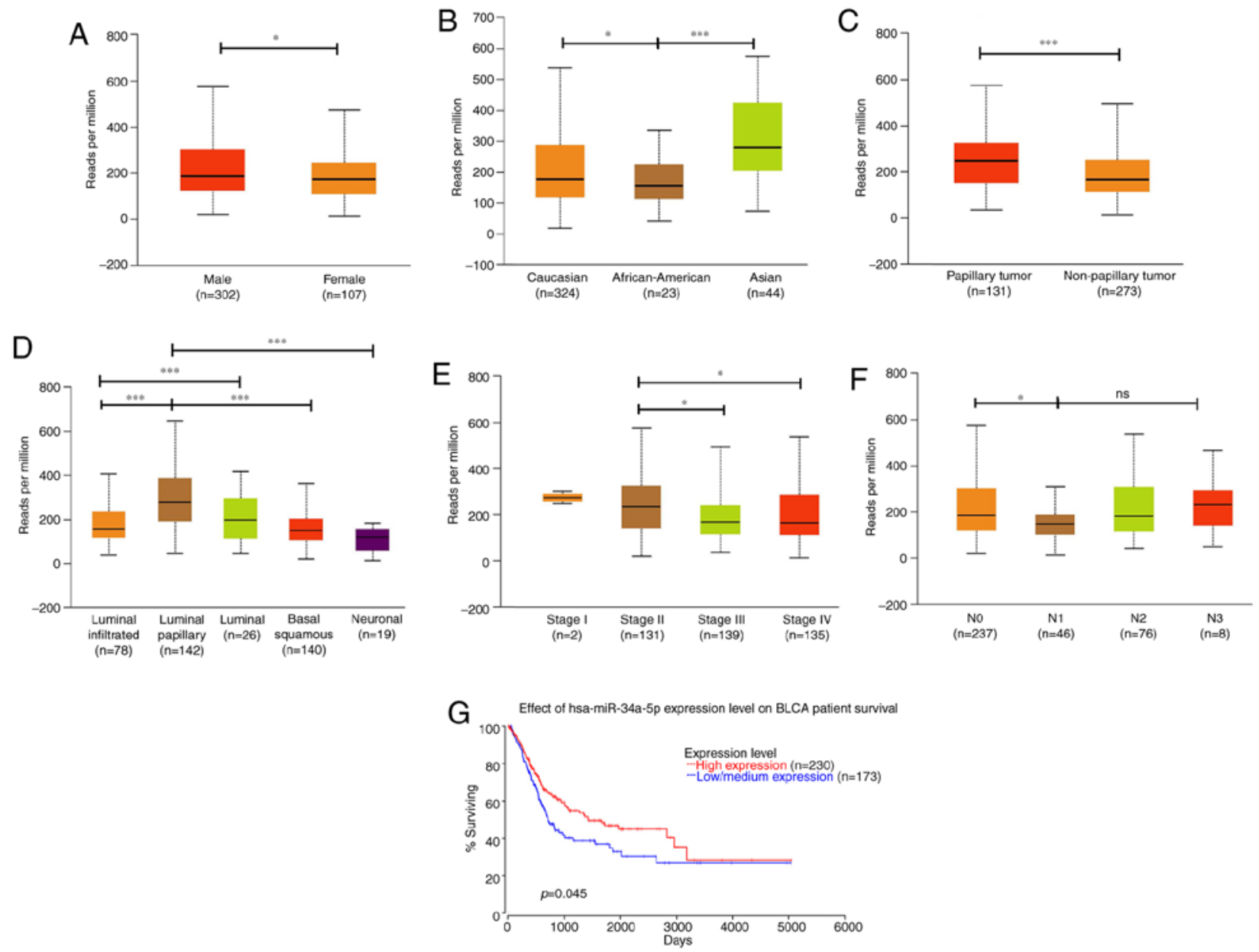

Figure 1. UALCAN portal analysis of BC samples based on the TCGA database. (A) Comparison of hsa-miR-34a expression between male and female individuals in BC samples. (B-F) Expression of hsa-miR-34a for different races, tumor histology, molecular subtypes, clinical stages, and nodal metastasis status of $\mathrm{BC}$ samples. (G) Overall survival rate between patients with $\mathrm{BC}$ with high and low hsa-miR-34a expression. All data are expressed as the mean \pm the $\mathrm{SD}$ of triplicate samples. ${ }^{*} \mathrm{P}<0.05,{ }^{* * * *} \mathrm{P}<0.001$ compared with the control group; ns, not significant. BC, bladder cancer; TCGA, The Cancer Genome Atlas,

matrix (BD Biosciences) for 30 min. The human BC cells in $200 \mu \mathrm{l}$ serum-free medium were seeded in Transwell inserts with a $300 \mu 1$ medium containing $1 \%$ FBS and placed in the lower chamber. Cell migration and invasion were imaged under x200 magnification by Eclipse Ti2 microscope (Nikon) after $24 \mathrm{~h}$.

Analysis of publicly available database. Using the TCGA dataset of bladder urothelial carcinoma, the correlations among MMP-2, hsa-mir-34a expression, race, tumor histology, molecular subtype, clinical stage, and nodal metastasis status were analyzed for each tumor sample through the UALCAN web server (http://ualcan.path.uab.edu/) (26). Overall survival rate between patients with $\mathrm{BC}$ with high and low hsa-miR-34a or MMP-2 expression was analyzed by OncoLnc (http://www.oncolnc.org/).

Statistical analysis. Statistical analyses were performed using SigmaPlot 10.0 (Systat Software, Inc.) and GraphPad Prism 7 (GraphPad Software. Inc.). The values are expressed as the mean \pm standard deviation (SD). All differences between experimental groups and controls were assessed for significance using Student's t-test. Between-group differences were considered to be significant if the P-value was $<0.05$.

\section{Results}

Clinical importance of hsa-miR-34a in human BC based on the TCGA Database. Numerous studies have classified hsa-mir-34a as a tumor suppressor because it inhibits tumorigenesis, and hsa-mir-34a is considered a potential therapeutic candidate for cancer (27). However, the clinical importance of hsa-mir-34a in human BC is largely unknown. We therefore analyzed the expression pattern of hsa-mir-34a by using different variables from The Cancer Genome Atlas (TCGA) Urothelial Bladder Carcinoma datasets through the UALCAN web server. Results revealed that hsa-miR-34a had a lower expression in female individuals diagnosed with $\mathrm{BC}(\mathrm{n}=107)$ than in male individuals $(n=302)(P=0.04628$; Fig. 1A). Notably, hsa-miR-34a expression was more enhanced in Asians $(n=44)$ than in Caucasians $(n=324 ; \mathrm{P}=0.0195)$ and African-Americans $(\mathrm{n}=23 ; \mathrm{P}=0.00007$; Fig. 1B). This result indicates that hsa-miR-34a expression varies according to genetic differences between races. We further analyzed the expression pattern of hsa-miR-34a in tumor histology and molecular subtypes of BC. Papillary urothelial carcinoma $(n=131)$, which progresses more slowly and has a more straightforward treatment and a more favorable prognosis than other types of BC (28), exhibited higher hsa-miR-34a expression than nonpapillary samples $(n=273 ; \mathrm{P}=0.00061$; 
A 5637

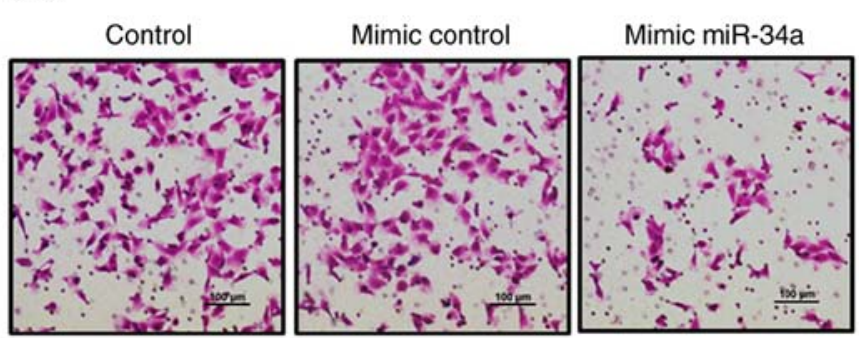

B UMUC3
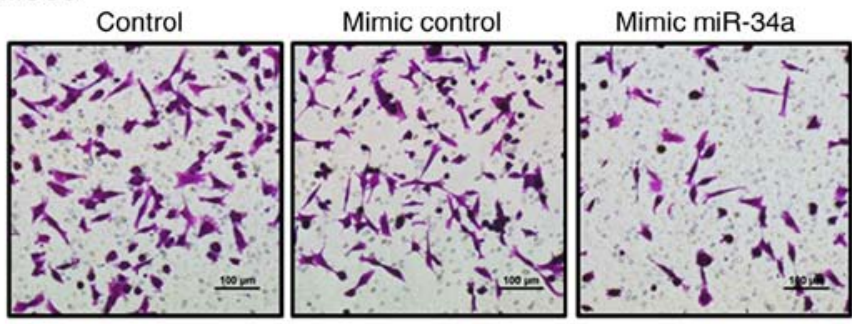

C 5637
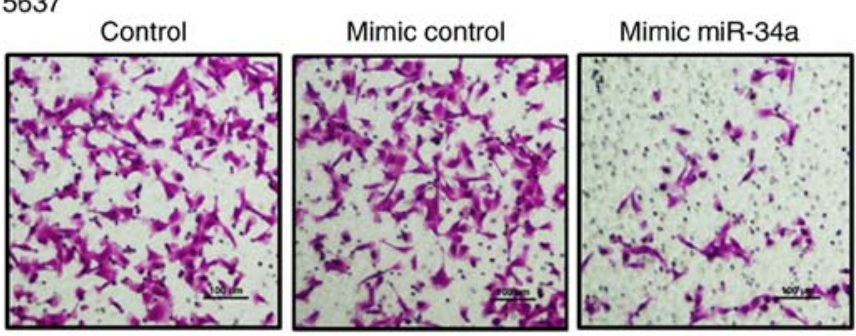

D UMUC3
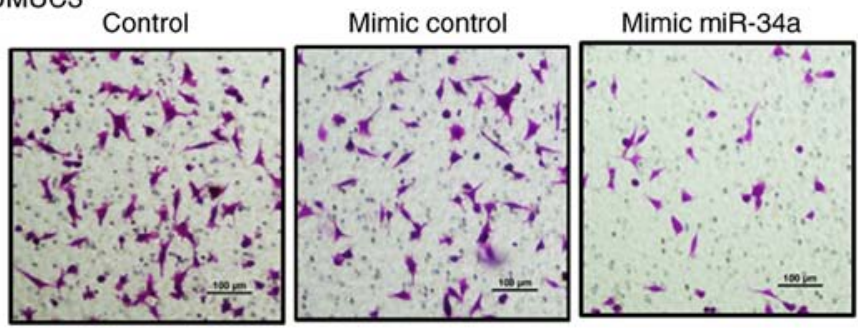
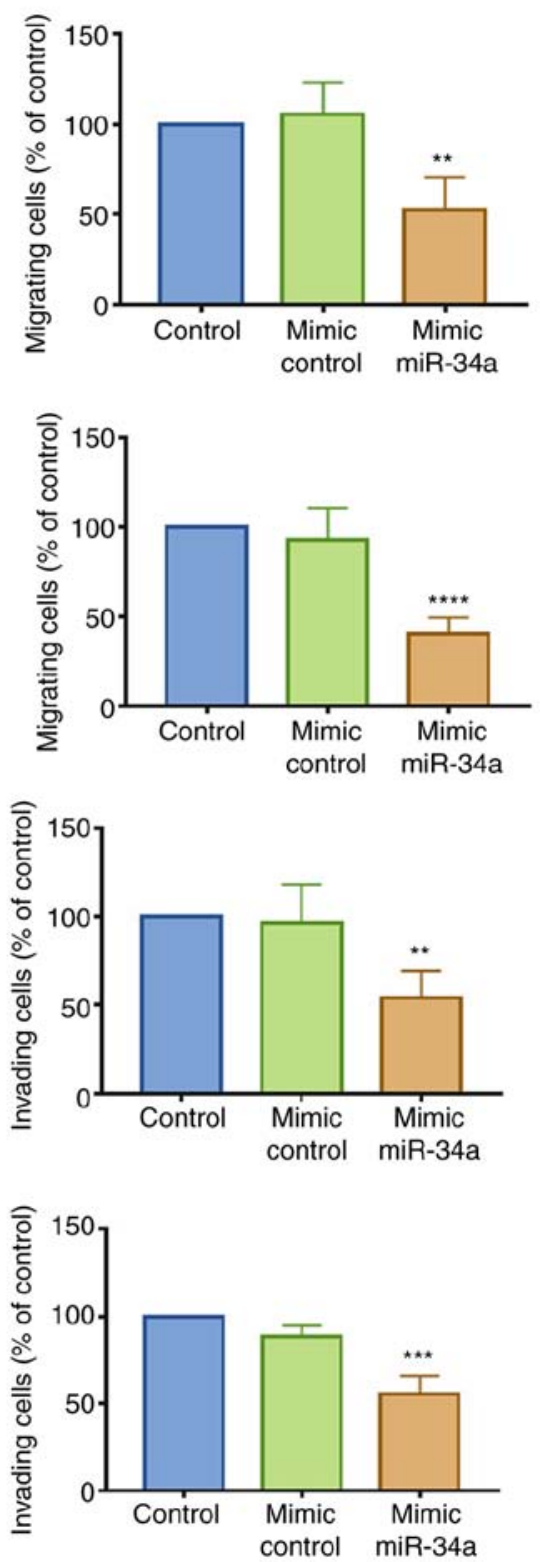

Figure 2. hsa-miR-34a affects the functions of cell migration and invasion in BC cells. (A-D) Cells were transfected with control or hsa-miR-34a mimic for $24 \mathrm{~h}$ after which cell migration and invasion were performed using Transwell assays. The number of migrated or invaded cells were quantified. All data are expressed as the mean $\pm \mathrm{SD}$ of triplicate samples. ${ }^{* *} \mathrm{P}<0.01,{ }^{* * *} \mathrm{P}<0.001,{ }^{* * * * *} \mathrm{P}<0.0001$ compared with the control group. BC, bladder cancer.

Fig. 1C). Moreover, MIBC has five molecular subtypes, namely luminal-papillary, luminal-infiltrated, luminal, basal-squamous, and neuronal (28). We found that the luminal-papillary subtype $(n=142)$ exhibited higher levels of hsa-miR-34a expression than did the other four molecular subtypes (Fig. 1D). By contrast, the neuronal subtype $(n=19)$, which has the poorest clinical outcome of all the subtypes, exhibited lower levels of hsa-miR-34a expression than did the luminal-papillary subtype $(\mathrm{P}=0.000002$; Fig. 1D). These data indicate that hsa-miR-34a may be positively associated with favorable clinical outcomes.

Next, we analyzed hsa-miR-34a expression in clinical stages and nodal metastasis status of BC samples. Results indicated that late stage (stage III and IV) had lower hsa-miR-34a expression than did early stage (stage II) tumors (stage II vs. III: $\mathrm{P}=0.025879$; stage II vs. IV: $\mathrm{P}=0.046764$; Fig. 1E). Moreover, significant associations were observed between low hsa-miR-34a expression levels and regional lymph node metastasis (N0 vs. N1: $\mathrm{P}=0.010535$; Fig. $1 \mathrm{~F}$ ). OncoLnc, a new TCGA data portal, focuses on survival correlations by using mRNA or miRNA expression data. Using OncoLnc analysis, we found that low hsa-miR-34a expression levels were significantly correlated with a low survival rate for patients with $\mathrm{BC}$ $(\mathrm{P}=0.045$; Fig. 1G). These data indicate that hsa-miR-34a, as a tumor suppressor, has significant associations with clinical and histopathologic characteristics in patients with BC.

hsa-miR-34a impedes cell migration and invasion in BC cells. hsa-miR-34a can inhibit cancer cell proliferation and migration (29). Therefore, we investigated whether hsa-miR-34a exhibits antitumor effects in inhibiting BC cell motility. An hsa-miR-34a mimic was used to analyze its function in regulating cell migration and invasion by employing a Transwell assay. We found that compared with the control mimic, hsa-miR-34a mimic significantly suppressed cell migration (Fig. 2A and B) 



Figure 3. hsa-miR-34a suppresses MMP-2 expression through direct binding. (A) An miRNA target prediction program (microRNA.org) was used to identify miRNAs that potentially bind to the MMP-2 3'-UTR. (B) Levels of MMP-2 mRNA expression in different BC cell lines were determined by qPCR assay. $(\mathrm{C}$ and D) Cells were cotransfected with wild-type or mutant MMP-2 3'-UTR $(1 \mu \mathrm{g} / \mu \mathrm{l})$ and control mimic or hsa-miR-34a mimic (100 nM) for $24 \mathrm{~h}$, and luciferase activities were measured. (E-G) Cells were transfected with control mimic or hsa-miR-34a mimic for $24 \mathrm{~h}$; the levels of MMP-2 protein and mRNA expression were measured using western blot analysis and qPCR assay, respectively. (H) Correlation between hsa-miR-34a and MMP-2 expression in BC samples. All data are expressed as the mean \pm the $\mathrm{SD}$ of triplicate samples. ${ }^{*} \mathrm{P}<0.05,{ }^{* * * *} \mathrm{P}<0.0001$ compared with the mimic control group. MMP-2, matrix metalloproteinase-2; BC, bladder cancer.

and invasion ability (Fig. 2C and D) in the 5637 and UMUC3 cell lines. The groups between the control and control mimic exhibited no such effects. The successful transfection of hsa-miR-34a mimic was confirmed (Fig. S1A). These data demonstrated that hsa-miR-34a reduced cell migration and invasion in BC cells.

hsa-miR-34a directly targets MMP-2. The action of miRNAs appears to involve mRNA silencing by binding to the 3'-UTRs (untranslated regions) at the miRNA recognition elements of target mRNAs (30). Subsequently, we investigated which target mRNA hsa-mir-34a binds to when impeding cell motility. On the basis of an analysis using the miRNA target prediction program (microRNA.org), we confirmed that hsa-miR-34a directly targets the 3'-UTR of MMP-2 (Fig. 3A). Therefore, we hypothesized that hsa-miR-34a affects BC cell migration and invasion through MMP-2 silencing. First, we assessed basal
MMP-2 expression levels in three BC cell lines. The 5637 and UMUC3 cells exhibited significantly higher MMP-2 mRNA and protein expression than T24 cells (Figs. 3B and S1B).

To confirm that hsa-miR-34a directly binds to the 3'-UTR of MMP-2 and inhibits MMP-2 mRNA translation, we constructed wild-type and mutant MMP-2-3'-UTR luciferase plasmids containing hsa-miR-34a binding sites. After transfection of 5637 and UMUC3 cells with wild-type luciferase plasmids, we found that the hsa-miR-34a mimic inhibited luciferase activity, but the mutant type had no such affect (Fig. 3C and D). Moreover, the hsa-miR-34a mimic diminished the levels of endogenous MMP-2 protein and mRNA expression in 5637 and UMUC3 cells (Fig. 3E-G). hsa-miR-34a expression was also negatively correlated with MMP-2 mRNA in human BC samples (Fig. 3H). According to our data, high hsa-miR-34a expression levels are associated 



Figure 4. Inhibition of MMP-2 expression suppresses cell motility in BC cells stably expressing hsa-miR-34a. (A and B) BC cells stably expressing control or hsa-miR-34a were established. The levels of MMP-2 protein and mRNA expression were determined using western blot analysis and qPCR assay, respectively. (C-F) Cell migration and invasion abilities in stable cells were determined using Transwell assays. The number of migrated or invaded cells were quantified. All data are expressed as the mean \pm SD of triplicate samples. ${ }^{* *} \mathrm{P}<0.01,{ }^{* * *} \mathrm{P}<0.001,{ }^{* * * * *} \mathrm{P}<0.0001$ compared with the control group. MMP-2, matrix metalloproteinase-2; BC, bladder cancer.

with improved clinical outcomes and significant suppression of MMP-2 expression by combining with the 3'-UTR region of MMP-2 mRNA.

Hsa-miR-34a regulates cell motility by suppressing MMP-2 expression. To confirm that MMP-2 is a critical mediator involved in hsa-miR-34a-regulated cell migration and invasion, BC cell lines 5637 and UMUC3 that stably expressed control or hsa-miR-34a were established. Low levels of MMP-2 expression were measured in hsa-miR-34a stable cells (Fig. 4A and B) and hsa-miR-34a inhibitor reversed the MMP-2 downregulation (Fig. S1C). The successful transfection of hsa-miR-34a inhibitor was verified (Fig. S1D). Moreover, hsa-mir-34a stable cells exhibited low mobility (Fig. 4C and D) and invasiveness (Fig. 4E and F). Thus, hsa-miR-34a regulates cell migration and invasion by suppressing MMP-2 expression in BC cells.

Clinicopathologic characteristics of MMP-2 in patients with $B C$. To further investigate the role of MMP-2 in human $\mathrm{BC}$, we analyzed the clinical importance of MMP-2 in human BC tissue samples derived from the Human Protein Atlas database. MMP-2 protein levels were higher in tumor specimens than in normal tissues (Fig. 5A and B). The data presented in Fig. 5C-H revealed that, in BC patients, MMP-2 expression differed according to sex, race, tumor histology, molecular subtype, tumor stages, and regional lymph node metastasis. Overexpression of MMP-2 was higher in Caucasians than in Asians ( $\mathrm{P}=0.012$; Fig. 5D). Similarly, MMP-2 expression was significantly elevated in $\mathrm{BC}$ tissues of nonpapillary $(\mathrm{P}=0.0043$; Fig. 5E) or luminal-infiltrated subtypes (luminal-infiltrated vs. luminal-papillary: $\mathrm{P}=1.76 \mathrm{E}-12$; Fig. 5F). Additionally, the mRNA levels of MMP-2 in patients with stage III and IV $\mathrm{BC}$ were clearly higher than those in patients with stage II (stage II vs. stage III: $\mathrm{P}=0.00002$; stage II vs. stage IV: $\mathrm{P}=0.00001$; Fig. 5G). We also found that high levels of MMP-2 expression were significantly correlated with low survival rates for patients with $\mathrm{BC}(\mathrm{P}=0.045$; Fig. 5I). However, no relationship was observed between MMP-2 expression and other clinical features such as sex (Fig. 5C) and regional lymph node metastasis (Fig. 5H). Taken together, our results indicate that increased MMP-2 might predict a poor prognosis for patients with BC, and MMP-2 is an excellent target for developing BC treatments.

\section{Discussion}

Bladder cancer (BC) is a common urologic cancer (31). Surgery and chemotherapy are useful standard treatments; however, tumor recurrence rates (an estimated 70\% within 5 years) remain high, and progression to invasive disease is extremely common (32). The poor prognosis in patients with $\mathrm{BC}$ is related to high local recurrence rates and distant metastases (33). Developing a novel and safer BC treatment that improves patient outcomes and recurrence rates is essential. This study investigated the therapeutic potential of hsa-miR-34a in inhibiting BC cell migration and invasion. 

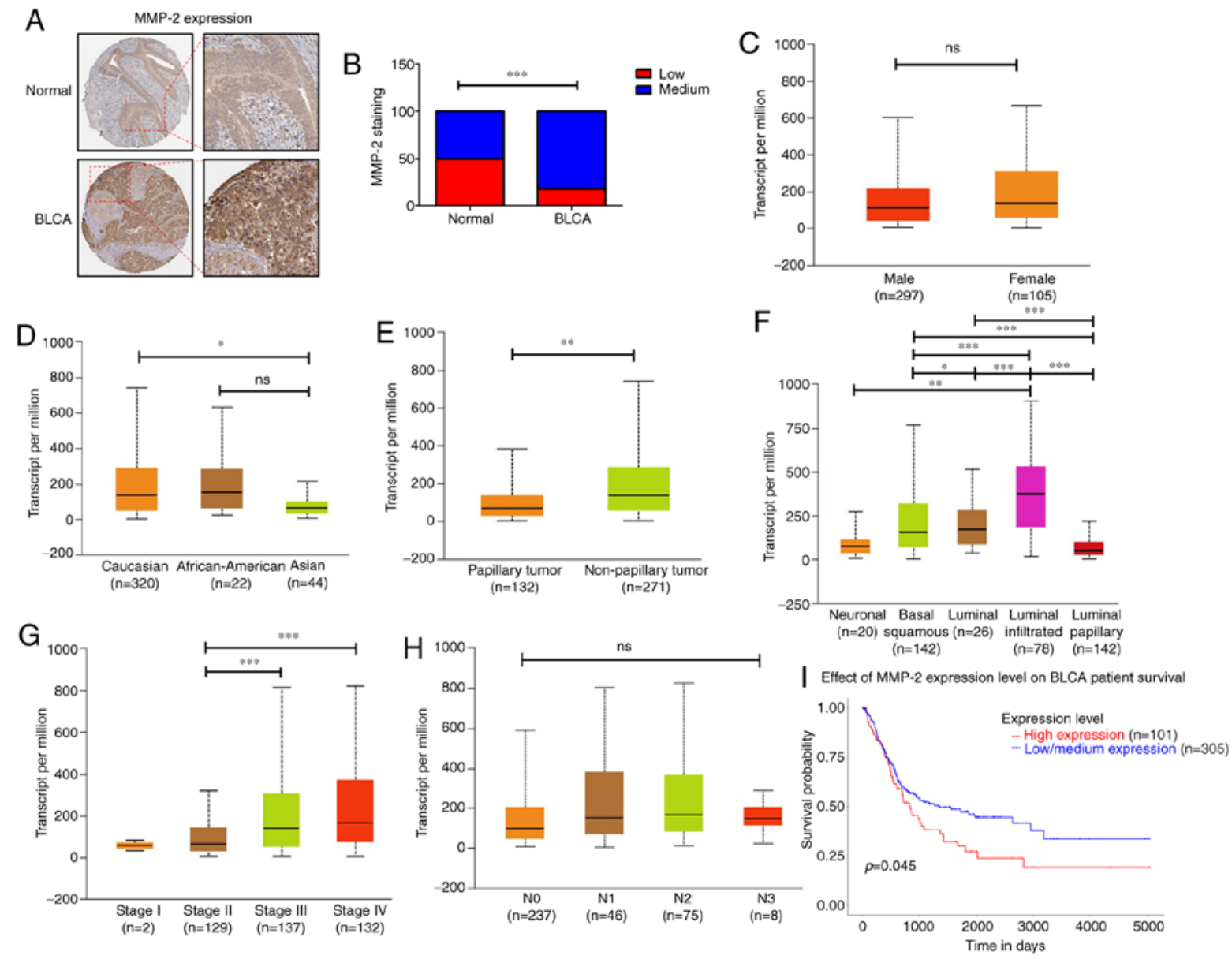

Figure 5. Levels of MMP-2 expression correlate with clinicopathologic features of BC. (A and B) Representative immunohistochemistry images of MMP-2 protein expression in BC tissues and normal bladder tissues. MMP-2 staining intensity was quantified. (C) Comparison of MMP-2 expression between male and female individuals in BC samples. (D-H) MMP-2 expression for different race, tumor histology, molecular subtypes, clinical stages, and nodal metastasis status of BC samples. (I) Survival probability between patients with BC with high and low MMP-2 expression. All data are expressed as the mean \pm SD of triplicate samples. ${ }^{*} \mathrm{P}<0.05,{ }^{* *} \mathrm{P}<0.01,{ }^{* * *} \mathrm{P}<0.001$ compared with the control group; ns, not significant. MMP-2, matrix metalloproteinase-2; $\mathrm{BC}$, bladder cancer.

The extracellular matrix (ECM) in the tumor microenvironment plays a vital role in regulating cancer progression (34). Notably, MMPs are involved in every step of tumor progression, such as promoting angiogenesis (35), tumor growth, and distant metastasis (36). MMP-14 silencing significantly reduced tumor cell migration and invasion ability (37). Similarly, MMP-9 silencing was found to diminish cell migration and invasion in glioma cells (38). Furthermore, in vivo analysis revealed that increased MMP-2 levels are correlated with melanoma progression (39). MMP-9 knockdown significantly inhibited tumor growth and metastasis in a mouse xenograft model of triple-negative breast cancer (40). In the present study, we found that high MMP-2 expression levels were significantly correlated with low survival rates in patients with BC. The mRNA levels of MMP-2 in patients with stage III and IV BC were markedly higher than those in patients with stage II. We further demonstrated that MMP-2 mediates BC cell migration and invasion. From a clinical perspective, MMP expression is correlated with tumor aggressiveness, disease stage, and poor prognosis in patients with cancers $(41,42)$. Together, these findings indicate that MMPs are an attractive target for cancer therapeutics. Cancer clinical trials have been conducted with numerous MMP inhibitors, including peptidomimetics, nonpeptidomimetics inhibitors, and tetracycline derivatives, which target MMPs in the extracellular space (43). However, the results have been disappointing and associated complications, such as musculoskeletal pain and inflammation, have been of great concern (43). Therefore, new drugs for the treatment of BC should be developed.

As crucial modulators in cellular pathways, microRNAs are instrumental in tumorigenesis and metastasis by targeting numerous oncogenes simultaneously through translation repression or mRNA degradation (44). A thorough understanding of associated downstream and upstream miRNAs is essential for successful treatment. In BC, hsa-miR-34a has been reported to attenuate metastasis and chemoresistance by reducing the levels of TCF1 and LEF1 (45). Also, hsa-miR-34a was found to inhibit $\mathrm{BC}$ cell migration and invasion by upregulating tumor-suppressor gene PTEN (46). Our study results concur with these findings, by showing that hsa-miR-34a inhibits MMP-2 expression and thereby suppresses cell migration and invasion in $\mathrm{BC}$ cells. The relevant results indicate that the hsa-miR-34a/MMP-2 axis is involved in the regulation of the antitumor effect of esophageal squamous cell carcinoma and glioma $(47,48)$. In addition, we also found that hsa-miR-34a was significantly correlated with clinical and histopathologic characteristics in human BC. However, hsa-miR-34a-mediated MMP-2 expression and tumor invasiveness should be evaluated 


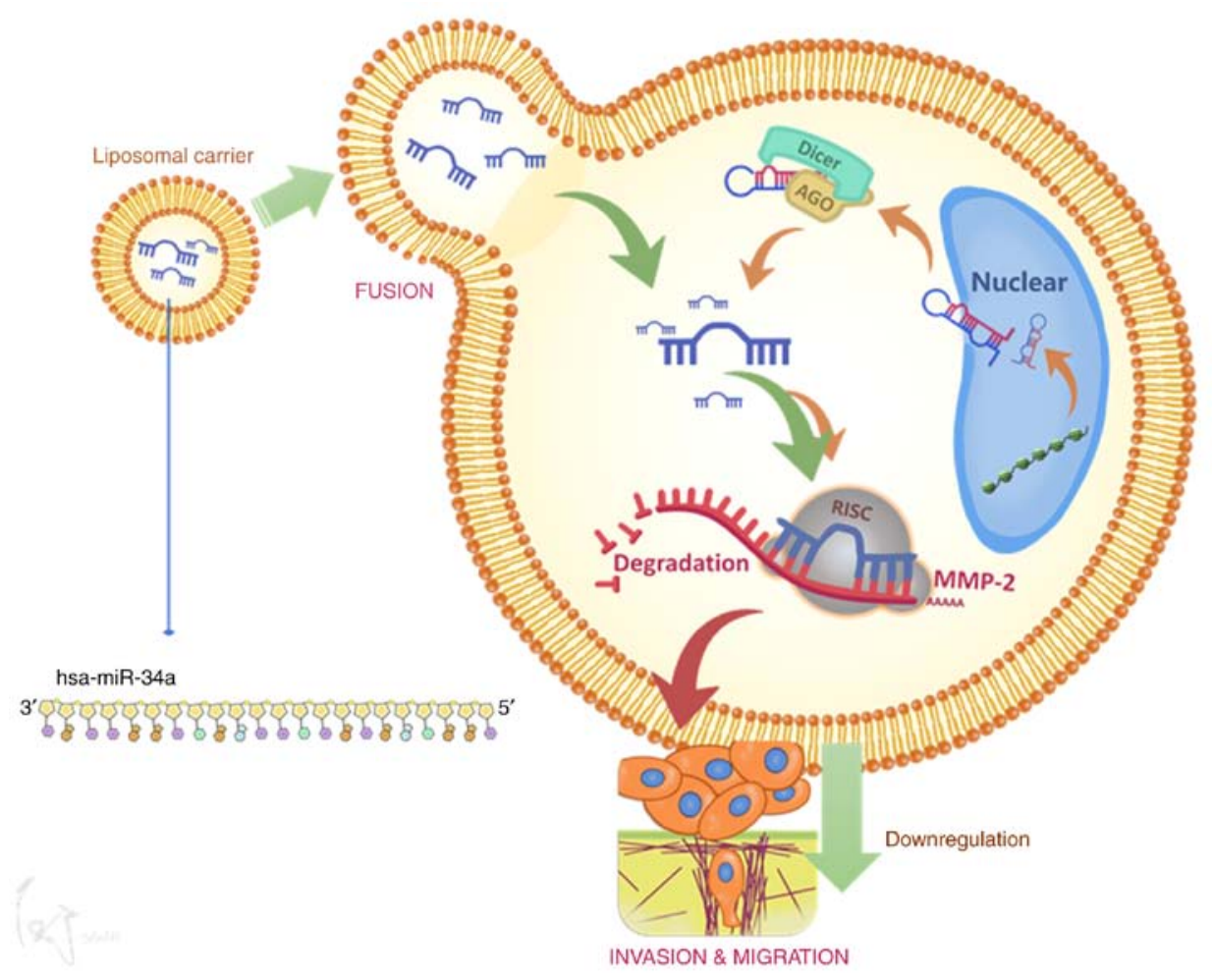

Figure 6. Schematic image depicting the mechanism by which hsa-miR-34a modulates cell motility through MMP-2 downregulation in BC cells. Modeling revealed that hsa-miR-34a directly targets the 3'-UTR of MMP-2 mRNA and thus suppresses MMP-2 translation. Overexpression of hsa-miR-34a in human $\mathrm{BC}$ cells may represent a potential treatment strategy. MMP-2, matrix metalloproteinase-2; BC, bladder cancer.

in vivo to confirm these findings. In multiple myeloma, intratumor or exogenous delivery of lipidic-formulated synthetic hsa-miR-34a mimic triggered antitumor action in an in vivo animal model (49,50). Related results revealed that systemic transfer of hsa-miR-34a mimic to tumors in an in vivo lung cancer animal model resulted in extraordinary suppression of tumor growth, suggesting the effectiveness of hsa-miR-34a replacement therapy in lung cancer $(51,52)$. Notably, MRX34, a liposomal formulation of nanoparticles packed with hsa-miR-34a mimics, is progressing to a phase I clinical trial (NCT01829971) for primary liver cancer, several solid tumors, and hematopoietic malignancies (27). Therefore, hsa-miR-34a is attractive as a potential treatment in cancers.

Although prior studies have emphasized the potential benefit of miRNA expression profiles in the prognosis for BC patients $(53,54)$, their value concerning ethnicity is unfamiliar. Caucasians are about twice as likely to develop BC as African-Americans, and Asians have slightly lower rates of BC (55). The reasons for these differences are not well understood. In the present investigation, we found that tumor-suppressor hsa-miR-34a has higher expression in Asians than Caucasians and African-Americans. This result suggests a racial-association for hsa-miR-34a in BC progression. hsa-miR-34a may repress tumorigenicity, especially in the Asian population, leading to a lower prevalence of BC. However, further investigation is still needed to prove the specificity of interaction between hsa-miR-34a and ethnicity.

In conclusion, hsa-miR-34a inhibits MMP-2 expression and thus suppresses cell migration and invasion (Fig. 6). These results may provide significant therapeutic opportunities for human BC.

\section{Acknowledgements}

We gratefully acknowledge the contributions of the TCGA Research Network: https://www.cancer.gov/tcga. We thank Jiun-Lin Lai for his preparation of the schematic summary of Fig. 6.

\section{Funding}

This work was supported by the Ministry of Science and Technology, Taiwan (MOST-108-2314-B-341-001-) and Shin Kong Wu Ho-Su Memorial Hospital (SKH-8302-106-DR-09).

\section{Availability of data and materials}

The datasets used during the present study are available from the corresponding author upon reasonable request.

\section{Authors' contributions}

Conceptualization of the study was achieved by KYC and TISH. Data curation was conducted by ACC and PCC. Formal analysis was carried out by TFT and YCL. Investigation of the data and results was conducted by KYC and ACC. Methodology was overseen by KYC. Project administration was carried out by HEC and CYH. Resources were provided by PCC and TFT. Software analysis was overseen by ACC and PCC. Supervision was carried out by CYH, YCL, HEC and TISH. Writing of the original draft was carried out by KYC and ACC. Writing of the review and editing were conducted by KYC and TISH. 


\section{Ethics approval and consent to participate}

Not applicable.

\section{Patient consent for publication}

Not applicable.

\section{Competing interests}

The authors of this article declare that they do not have any competing interests.

\section{References}

1. Siegel R, Naishadham D and Jemal A: Cancer statistics, 2012 CA Cancer J Clin 62: 10-29, 2012.

2. Antoni S, Ferlay J, Soerjomataram I, Znaor A, Jemal A and Bray F: Bladder cancer incidence and mortality: A global overview and recent trends. Eur Urol 71: 96-108, 2017.

3. Isharwal S and Konety B: Non-muscle invasive bladder cancer risk stratification. Indian J Urol 31: 289-296, 2015.

4. Sylvester RJ, van der Meijden AP, Oosterlinck W, Witjes JA Bouffioux C, Denis L, Newling DW and Kurth K: Predicting recurrence and progression in individual patients with stage Ta T1 bladder cancer using EORTC risk tables: A combined analysis of 2,596 patients from seven EORTC trials. Eur Urol 49: 466-465; Discussion 475-467, 2006.

5. Kobayashi T: Understanding the biology of urothelial cancer metastasis. Asian J Urol 3: 211-222, 2016.

6. van Zijl F, Krupitza G and Mikulits W: Initial steps of metastasis: Cell invasion and endothelial transmigration. Mutat Res 728 : 23-34, 2011.

7. Yadav L, Puri N, Rastogi V, Satpute P, Ahmad R and Kaur G: Matrix metalloproteinases and cancer-roles in threat and therapy. Asian Pac J Cancer Prev 15: 1085-1091, 2014.

8. Nannuru KC, Futakuchi M, Varney ML, Vincent TM, Marcusson EG and Singh RK: Matrix metalloproteinase (MMP)-13 regulates mammary tumor-induced osteolysis by activating MMP9 and transforming growth factor-beta signaling at the tumor-bone interface. Cancer Res 70: 3494-3504, 2010.

9. Verma S, Kesh K, Gupta A and Swarnakar S: An overview of matrix metalloproteinase 9 polymorphism and gastric cancer risk. Asian Pac J Cancer Prev 16: 7393-7400, 2015.

10. Malcherczyk D, Heyse TJ, El-Zayat BF, Kunzke V, Moll R, Fuchs-Winkelmann S and Paletta JRJ: Expression of MMP-9 decreases metastatic potential of Chondrosarcoma: An immunohistochemical study. BMC Musculoskelet Disord 19: 9, 2018.

11. Li H, Qiu Z, Li F and Wang C: The relationship between MMP-2 and MMP-9 expression levels with breast cancer incidence and prognosis. Oncol Lett 14: 5865-5870, 2017.

12. Reis ST, Leite KR, Piovesan LF, Pontes-Junior J, Viana NI, Abe DK, Crippa A, Moura CM, Adonias SP, Srougi M and Dall'Oglio MF: Increased expression of MMP-9 and IL-8 are correlated with poor prognosis of Bladder Cancer. BMC Urol 12: $18,2012$.

13. Zhou W, Yu X, Sun S, Zhang X, Yang W, Zhang J, Zhang X and Jiang Z: Increased expression of MMP-2 and MMP-9 indicates poor prognosis in glioma recurrence. Biomed Pharmacother 118: 109369, 2019.

14. Ranganathan K and Sivasankar V: MicroRNAs-Biology and clinical applications. J Oral Maxillofac Pathol 18: 229-234, 2014

15. Eichhorn SW, Guo H, McGeary SE, Rodriguez-Mias RA, Shin C, Baek D, Hsu SH, Ghoshal K, Villén J and Bartel DP: mRNA destabilization is the dominant effect of mammalian microRNAs by the time substantial repression ensues. Mol Cell 56: 104-115, 2014.

16. Hammond SM: MicroRNAs as oncogenes. Curr Opin Genet Dev 16: 4-9, 2006

17. Lodygin D, Tarasov V, Epanchintsev A, Berking C, Knyazeva T, Körner H, Knyazev P, Diebold J and Hermeking H: Inactivation of miR-34a by aberrant $\mathrm{CpG}$ methylation in multiple types of cancer. Cell Cycle 7: 2591-2600, 2008.

18. Chim CS, Wong KY, Qi Y, Loong F, Lam WL, Wong LG, Jin DY, Costello JF and Liang R: Epigenetic inactivation of the miR-34a in hematological malignancies. Carcinogenesis 31: 745-750, 2010.
19. Liu C, Kelnar K, Liu B, Chen X, Calhoun-Davis T, Li H, Patrawala L, Yan H, Jeter C, Honorio S, et al: The microRNA miR-34a inhibits prostate cancer stem cells and metastasis by directly repressing CD44. Nat Med 17: 211-215, 2011.

20. Zhang C, Yao Z, Zhu M, Ma X, Shi T, Li H, Wang B, Ouyang J and Zhang X: Inhibitory effects of microRNA-34a on cell migration and invasion of invasive urothelial bladder carcinoma by targeting Notch1. J Huazhong Univ Sci Technolog Med Sci 32: 375-382, 2012

21. Yu G, Yao W, Xiao W, Li H, Xu H and Lang B: MicroRNA-34a functions as an anti-metastatic microRNA and suppresses angiogenesis in bladder cancer by directly targeting CD44. J Exp Clin Cancer Res 33: 779, 2014.

22. Zhou J, Zhou W, Kong F, Xiao X, Kuang H and Zhu Y: microRNA-34a overexpression inhibits cell migration and invasion via regulating SIRT1 in hepatocellular carcinoma. Oncol Lett 14: 6950-6954, 2017.

23. Yang S, Li Y, Gao J, Zhang T, Li S, Luo A, Chen H, Ding F, Wang X and Liu Z: MicroRNA-34 suppresses breast cancer invasion and metastasis by directly targeting Fra-1. Oncogene 32 : 4294-4303, 2013

24. Lin CJ, Gong HY, Tseng HC, Wang WL and Wu JL: miR-122 targets an anti-apoptotic gene, Bcl-w, in human hepatocellular carcinoma cell lines. Biochem Biophys Res Commun 375: 315-320, 2008.

25. Lin JF, Lin YC, Yang SC, Tsai TF, Chen HE, Chou KY and Hwang TI: Autophagy inhibition enhances RAD001-induced cytotoxicity in human bladder cancer cells. Drug Des Devel Ther 10: 1501-1513, 2016.

26. Chandrashekar DS, Bashel B, Balasubramanya SAH, Creighton CJ, Ponce-Rodriguez I, Chakravarthi BVSK and Varambally S: UALCAN: A portal for facilitating tumor subgroup gene expression and survival analyses. Neoplasia 19: 649-658, 2017.

27. Zhang L, Liao Y and Tang L: MicroRNA-34 family: A potential tumor suppressor and therapeutic candidate in cancer. J Exp Clin Cancer Res 38: 53, 2019.

28. Inamura K: Bladder cancer: New insights into its molecular pathology. Cancers (Basel) 10: 100, 2018.

29. Li L, Yuan L, Luo J, Gao J, Guo J and Xie X: MiR-34a inhibits proliferation and migration of breast cancer through downregulation of Bcl-2 and SIRT1. Clin Exp Med 13: 109-117, 2013.

30. Bartel DP: MicroRNAs: Genomics, biogenesis, mechanism, and function. Cell 116: 281-297, 2004.

31. Yaxley JP: Urinary tract cancers: An overview for general practice. J Family Med Prim Care 5: 533-538, 2016.

32. Shen Z, Shen T, Wientjes MG, O'Donnell MA and Au JL: Intravesical treatments of bladder cancer: Review. Pharm Res 25: $1500-1510,2008$

33. Di Pierro GB, Gulia C, Cristini C, Fraietta G, Marini L, Grande P Gentile V and Piergentili R: Bladder cancer: A simple model becomes complex. Curr Genomics 13: 395-415, 2012.

34. Zhu J, Xiong G, Trinkle C and Xu R: Integrated extracellular matrix signaling in mammary gland development and breast cancer progression. Histol Histopathol 29: 1083-1092, 2014.

35. Quintero-Fabián S, Arreola R, Becerril-Villanueva E, Torres-Romero JC, Arana-Argáez V, Lara-Riegos J, Ramírez-Camacho MA and Alvarez-Sánchez ME: Role of matrix metalloproteinases in angiogenesis and cancer. Front Oncol 9: 1370, 2019

36. Kessenbrock K, Plaks V and Werb Z: Matrix metalloproteinases: Regulators of the tumor microenvironment. Cell 141: 52-67,2010.

37. Ueda J,Kajita M,Suenaga N,Fujii Kand Seiki M: Sequence-specific silencing of MT1-MMP expression suppresses tumor cell migration and invasion: Importance of MT1-MMP as a therapeutic target for invasive tumors. Oncogene 22: 8716-8722, 2003.

38. Lakka SS, Rajan M, Gondi C, Yanamandra N, Chandrasekar N, Jasti SL, Adachi Y, Siddique K, Gujrati M, Olivero W, et al: Adenovirus-mediated expression of antisense MMP-9 in glioma cells inhibits tumor growth and invasion. Oncogene 21: 8011-8019, 2002.

39. Hofmann UB, Westphal JR, Zendman AJ, Becker JC, Ruiter DJ and van Muijen GN: Expression and activation of matrix metalloproteinase-2 (MMP-2) and its co-localization with membrane-type 1 matrix metalloproteinase (MT1-MMP) correlate with melanoma progression. J Pathol 191: 245-256, 2000.

40. Mehner C, Hockla A,Miller E, Ran S, Radisky DC and Radisky ES: Tumor cell-produced matrix metalloproteinase 9 (MMP-9) drives malignant progression and metastasis of basal-like triple negative breast cancer. Oncotarget 5: 2736-2749, 2014. 
41. Vihinen P and Kähäri VM: Matrix metalloproteinases in cancer: Prognostic markers and therapeutic targets. Int J Cancer 99: 157-166, 2002.

42. Itoh T, Tanioka M, Yoshida $\mathrm{H}$, Yoshioka $\mathrm{T}$, Nishimoto $\mathrm{H}$ and Itohara S: Reduced angiogenesis and tumor progression in gelatinase A-deficient mice. Cancer Res 58: 1048-1051, 1998.

43. Gialeli C, Theocharis AD and Karamanos NK: Roles of matrix metalloproteinases in cancer progression and their pharmacological targeting. FEBS J 278: 16-27, 2011.

44. Skaftnesmo KO, Prestegarden L, Micklem DR and Lorens JB: MicroRNAs in tumorigenesis. Curr Pharm Biotechnol 8: 320-325, 2007

45. Liu X, Liu X, Wu Y, Fang Z, Wu Q, Wu C, Hao Y, Yang X, Zhao J, Li J, et al: MicroRNA-34a attenuates metastasis and chemoresistance of bladder cancer cells by targeting the TCF1/LEF1 axis Cell Physiol Biochem 48: 87-98, 2018.

46. Ding ZS, He YH, Deng YS, Peng PX, Wang JF, Chen X, Zhao PY and Zhou XF: MicroRNA-34a inhibits bladder cancer cell migration and invasion, and upregulates PTEN expression. Oncol Lett 18: 5549-5554, 2019.

47. Yang L, Song X, Zhu J, Li M, Ji Y, Wu F, Chen Y, Cui X, Hu J, Wang L, et al: Tumor suppressor microRNA-34a inhibits cell migration and invasion by targeting MMP-2/MMP-9/FNDC3B in esophageal squamous cell carcinoma. Int J Oncol 51: 378-388, 2017.

48. Zhao H, Xing F, Yuan J, Li Z and Zhang W: Sevoflurane inhibits migration and invasion of glioma cells via regulating miR-34a-5p/MMP-2 axis. Life Sci 256: 117897, 2020.

49. Di Martino MT, Leone E, Amodio N, Foresta U, Lionetti M, Pitari MR, Cantafio ME, Gullà A, Conforti F, Morelli E, et al: Synthetic miR-34a mimics as a novel therapeutic agent for multiple myeloma: In vitro and in vivo evidence. Clin Cancer Res 18: 6260-6270, 2012.
50. Yan D, Zhou X, Chen X, Hu DN, Dong XD, Wang J, Lu F, Tu L and Qu J: MicroRNA-34a inhibits uveal melanoma cell proliferation and migration through downregulation of c-Met. Invest Ophthalmol Vis Sci 50: 1559-1565, 2009.

51. Wiggins JF, Ruffino L, Kelnar K, Omotola M, Patrawala L, Brown D and Bader AG: Development of a lung cancer therapeutic based on the tumor suppressor microRNA-34. Cancer Res 70: 5923-5930, 2010.

52. Trang P, Wiggins JF, Daige CL, Cho C, Omotola M, Brown D, Weidhaas JB, Bader AG and Slack FJ: Systemic delivery of tumor suppressor microRNA mimics using a neutral lipid emulsion inhibits lung tumors in mice. Mol Ther 19: 1116-1122, 2011.

53. Dyrskjøt L, Ostenfeld MS, Bramsen JB, Silahtaroglu AN, Lamy P, Ramanathan R, Fristrup N, Jensen JL, Andersen CL, Zieger K, et al: Genomic profiling of microRNAs in bladder cancer: miR-129 is associated with poor outcome and promotes cell death in vitro. Cancer Res 69: 4851-4860, 2009.

54. Xie Y, Ma X, Chen L, Li H, Gu L, Gao Y, Zhang Y, Li X, Fan Y, Chen J and Zhang X: MicroRNAs with prognostic significance in bladder cancer: A systematic review and meta-analysis. Sci Rep 7: 5619, 2017.

55. Al-Husseini MJ, Kunbaz A, Saad AM, Santos JV, Salahia S, Iqbal $\mathrm{M}$ and Alahdab F: Trends in the incidence and mortality of transitional cell carcinoma of the bladder for the last four decades in the USA: A SEER-based analysis. BMC Cancer 19: 46, 2019.

(7) (\$) This work is licensed under a Creative Commons Attribution-NonCommercial 4.0 International (CC BY-NC 4.0) License. 